\title{
Visual Abnormalities Associate With Hippocampus in Mild Cognitive Impairment and Early Alzheimer's Disease
}

\begin{abstract}
Aonan Zhao ${ }^{1 \dagger}$, Fang Fang ${ }^{2 \dagger}$, Binyin $\mathrm{Li}^{1}$, Yan $\mathrm{Chen}^{3}$, Yinghui Qiu ${ }^{1}$, Yanli $\mathrm{Wu}^{3}$, Wei $\mathrm{Xu}^{1 *}$ and Yulei Deng ${ }^{1,4 *}$

${ }^{1}$ Department of Neurology, Institute of Neurology, Ruijin Hospital, Shanghai Jiao Tong University School of Medicine, Shanghai, China, ${ }^{2}$ Department of Geriatrics, Ruijin Hospital, Shanghai Jiao Tong University School of Medicine, Shanghai, China, ${ }^{3}$ Department of Ophthalmology, Ruijin Hospital, Shanghai Jiao Tong University School of Medicine, Shanghai, China, ${ }^{4}$ Department of Neurology, Ruijin Hospital, Luwan Branch, Shanghai Jiao Tong University School of Medicine, Shanghai, China
\end{abstract}

Background and Objective: Alzheimer's disease (AD) has been shown to affect vision in human patients and animal models. This study was conducted to explore ocular abnormalities in the primary visual pathway and their relationship with hippocampal atrophy in patients with $A D$ and mild cognitive impairment $(\mathrm{MCl})$. The aim of this study was to investigate the potential value of ocular examinations as a biomarker during the AD progression.

Methods: Patients with $\mathrm{MCl}(n=23)$ or $\mathrm{AD}(n=17)$ and age-matched cognitively normal controls (NC; $n=19$ ) were enrolled. Pattern visual-evoked potentials (PVEP), flash electroretinogram (FERG) recordings and optical coherence tomography (OCT) were performed for all participants. Hippocampal volumes were measured by 3T magnetic resonance imaging. Cognitive function was assessed by Mini Mental State Examination (MMSE), Montreal Cognitive Assessment (MoCA) and Alzheimer's Disease Assessment Scale-cognitive subscale (ADAS-cog). Pearson correlation was employed to analyze the potential associations between ocular abnormalities and hippocampal volumes. Hierarchical regression models were conducted to determine associations between cognitive performances and ocular abnormalities as well as hippocampal volumes after adjusting for confounding factors including age, sex, cognitive reserve, and APOE4 status.

Results: PVEP amplitude of P100 waveform was significantly decreased in AD patients compared to $\mathrm{MCl}$ and normal individuals. In FERG test, delayed latencies of rod response, rod cone response and 3.0 flicker time were found in cognitively impaired groups, indicating dysfunctions of both the rod and cone systems in the disease progression. OCT test revealed reduced macular retinal nerve fiber layer (m-RNFL) thickness in $\mathrm{MCl}$ and $\mathrm{AD}$ patients, which significantly correlated with brain structure of hippocampus particularly vulnerable during the progression of $A D$. Interestingly, P100 amplitude showed a significant association with hippocampal volumes even after adjusting confounding factors including age, sex, and cognitive reserve. Hierarchical 
regression analysis further demonstrated that $m-R N F L$ thickness, as well as hippocampal volumes, significantly associated with ADAS-cog scores.

Conclusion: P100 amplitude and m-RNFL thickness showed significant correlations with brain structure involved in AD-related neurodegeneration, and therefore proved to be potential indicators of brain imaging pathologies.

Keywords: Alzheimer's disease, mild cognitive impairment, hippocampus, visual abnormalities, P100 amplitude, m-RNFL thickness

\section{INTRODUCTION}

Alzheimer's disease $(\mathrm{AD})$ is the most common cause of degenerative dementia in older people (Livingston et al., 2020). The early symptoms begin with short-term memory loss and gradually progress to severe impairment in memory, thinking and behavior (Chan et al., 2013). Mild cognitive impairment $(\mathrm{MCI})$ is defined as an early stage of dementia and more than $50 \%$ of $\mathrm{MCI}$ patients progress to $\mathrm{AD}$ in a period of 4-5 years (Petersen, 2006). Therefore, screening, diagnosis and targeted treatment in this stage to prevent conversion to $\mathrm{AD}$ is of great importance. Various studies have attempted to identify and evaluate biomarkers for $\mathrm{AD}$ and $\mathrm{MCI}$, including state-of-the-art neuroimaging techniques and biochemical analysis of the cerebrospinal fluid. However, timeconsuming, radiation, high cost, and invasiveness have hampered their widespread availability.

In recent years, ocular tests have received substantial attention from the scientific community for ocular structural and functional changes in $\mathrm{AD}$ through non-invasive and inexpensive evaluation (Heaton et al., 2015). Lots of evidence indicates that visual disturbance is common for AD patients (Cormack et al., 2000). In some AD patients, visual disturbances present as the initial complaint, such as impairment in visual acuity, spatial contrast sensitivity, color sensitivity, and blurred vision (Croningolomb, 1995; Armstrong, 1996). Quantitative data supports the assessment that in the primary visual pathway including retina and lens amyloid-beta accumulation, retinal nerve fiber layer loss, and visual cortex changes can be valuable for the diagnosis of $\mathrm{AD}$ (Goldstein et al., 2003; Ohno-Matsui, 2011; Ikram et al., 2012). Optical coherence tomography (OCT) is a non-invasive imaging technique that captures high-resolution and three-dimensional images of the retina. Previous studies in $\mathrm{AD}$ patients demonstrated that the retinal nerve fiber layer (RNFL) thickness was attenuated in comparison to healthy controls (Iseri et al., 2006). In addition, the decrease in parapapillary and macular RNFL thickness, and macular volume were related to the cognitive ability of $\mathrm{AD}$ patients (Ikram et al., 2012). Other than structural measures, flash electroretinogram (FERG), and pattern visual evoked potential (PVEP) are classic

\footnotetext{
Abbreviations: AD, Alzheimer's disease; MCI, Mild cognitive impairment; PVEP, Pattern visual-evoked potentials; FERG, Flash electroretinogram; OCT, Optical coherence tomography; MMSE, Mini Mental State Examination; MoCA, Montreal Cognitive Assessment; ADAS-cog, Alzheimer's Disease Assessment Scale-cognitive subscale; m-RNFL, Macular retinal nerve fiber layer; SDS, Self-rating depression scale; SAS, Self-rating anxiety scale; MRI, Magnetic resonance imaging.
}

tests to assess full retinal function and retinal ganglion cells, respectively. Abnormal changes are reported in FERG and PVEP in early AD patients as well (Krasodomska et al., 2010).

In this study, we aim to perform ophthalmological examinations including PVEP, FERG and OCT tests to explore ocular functional and structural changes in patients with $\mathrm{AD}$ or MCI and age-matched cognitively normal individuals. Since the hippocampus is well-known to be involved in neurodegenerative processes and atrophy of hippocampus predicts the conversion from MCI to AD (Erten-Lyons et al., 2006), we investigated hippocampal volumes by MRI and attempt to identify the relationship between hippocampal volumes and visual system impairment. Our research's goal was to evaluate the potential availability of the application of non-invasive, cost-effective ophthalmic screening tests for AD.

\section{MATERIALS AND METHODS}

\section{Patients Enrolment}

Participants $(N=59)$ in this study were enrolled at the neurology clinic of Ruijin Hospital affiliated to the Shanghai Jiao Tong University School of Medicine, Shanghai, China, from September 2016 to December 2020. All volunteers gave their informed, written consent prior to study participation. This study was approved by the Research Ethics Committee of Ruijin Hospital. All patients with $\mathrm{AD}$ dementia were diagnosed as probable $\mathrm{AD}$ dementia following the National Institute on Aging and Alzheimer's Association (NIA-AA) diagnostic guidelines for probable $\mathrm{AD}$ dementia with support of structural MRI images (McKhann et al., 2011). To ensure volunteers understood the task, only patients with mild to moderate AD dementia [ $24 \geq$ Mini Mental State Examination $(\mathrm{MMSE}) \geq 10$ ] participated on the tests. MCI with deficits in memory function were diagnosed according to the Mayo Clinic criteria (Petersen, 2004). The criteria include subjective memory complaint corroborated by an informant together with preserved everyday activities, a memory impairment based on a standard neuropsychological test, preserved global cognitive functions and finally the exclusion of dementia. Age- and education-adjusted scores falling 1.5 standard deviations below that expected for age and education level may indicate MCI but these are considered as guidelines rather than diagnostic cut-offs. The cognitively normal participants were age-, sex-, and education-matched and were recruited from the local community in Shanghai. Inclusion criteria for normal controls required a MMSE score $\geq 28$ without any memory-related complaint. Participants with the presence 
of dementia or other neurological diseases such as Parkinson's disease were excluded. Besides, individuals were excluded if they have any of the following medical problems: acute diabetic complications, history of acute cerebrovascular accident, history of acute cardiovascular accident, systemic disorders such as malignancy and lupus which were not cured, severe infection, drug abuse or dependency condition and severe psychiatric disorders which were not cured. Individuals with any ocular disease, high refractive error, systemic disease affecting vision or history of ophthalmic surgery were excluded.

\section{Neuropsychological Assessment}

Each participant received a detailed neuropsychological assessment by a memory-related specialist. Additionally, patients with vascular dementia, mental disorders, other neurological diseases, and history of alcohol or drug abuse were also excluded. Specialists assessed cognitive ability in all participants utilizing Mini-Mental State Examination (MMSE, developed by Zhang et al., 1990), Montreal Cognitive Assessment (MoCA) Beijing version, Self-rating depression scale (SDS), Self-rating anxiety scale (SAS), and the 12-item Chinese version of Alzheimer's Disease Assessment Scale-cognitive subscale (ADAS-cog). The cut point established for the MMSE that defines "Alzheimer's disease" was set at 24 (Lopez et al., 2005). We used MoCA cut-off scores of $\leq 24$ points for MCI and $\leq 22$ points for dementia and for individual within 6 years or fewer of formal education, one point was added to the score as a correction (Goldstein et al., 2014). The clinical cut-off raw scores for SAS and SDS were set at 36 and 40, respectively (Dunstan and Scott, 2018). The optimal cut-off score for ADAS-Cog 12-item scale to discriminate between MCI and mild AD was $\geq 21$ (Zainal et al., 2016).

\section{Optical Coherence Tomography}

All participants underwent the OCT examination using the STRATUS OCT Model 3000 (Carl Zeiss Meditec, Inc., Dublin, California, USA). The retinal mapping software was used to calculate the average retinal thickness of the central ring. All eyes were scanned in a radial-spoked pattern centered on the foveola with the scan length of $6 \mathrm{~mm}$. The mean and standard deviations of the macular RNFL (m-RNFL) were calculated in superior, inferior hemiretina. These measurements were performed out to one disc diameter inferiorly and superiorly to the fovea. Optic disc head scans were recorded with optimized z-offset and polarization. The Fast Optic Disc Scan Protocol (OCT-DISC) was used. The software determined automatically the disc margin setting a point at the edge of the retinal pigment epithelium (RPE)/choriocapillaris layer on each side of the disc along a crosssection. The operator was permanently monitoring for steady eye fixation, correct scan position, and good signal-to-noise ratio. Scans were repeated until an image of satisfying quality was obtained.

\section{Pattern Visual Evoked Potential}

All the PVEP examinations (UTAS-E3000, LKC Technologies Inc., Gaithersburg, USA) were performed in an electrically shielded room. Cup-shaped electrodes of $\mathrm{Ag} / \mathrm{AgCl}$ were placed according to the International 10/20 system (Odom et al., 2004) at the following positions: active electrode in $\mathrm{Oz}$, reference electrode in $\mathrm{Fz}$, ground electrode on earlobes. The recordings were performed in a quiet and dimmed room. The electrical potentials of the occipital cortex were recorded while the participant was looking at the fixation point in the middle of the moving checkboard patterns on the screen one meter in front. The luminance of the white areas was $80 \mathrm{~cd} \mathrm{~m}^{-2}$, and the contrast between the checks was $100 \%$. The checkerboard pattern reversed at the rate of $2 \mathrm{~Hz}$ and the viewing distance was $100 \mathrm{~cm}$, the check edges subtended at $15^{\prime}$ of visual angle. The signals were fed into an amplifier with the low frequency cutoff filter set at $1.0 \mathrm{~Hz}$ and the high frequency cut-off filter set at $100 \mathrm{~Hz}$. The amplified responses were fed to a signal-averaging computer, and 100 responses were averaged with an analysis time of $500 \mathrm{~ms}$. The impedance was kept below $5 \mathrm{kOhm}$. An alternant checkboard pattern was used as a stimulating pattern and the check size was $23 \mathrm{~min}$. There are three separate phases in the VEP waveform: an initial negative deflection (N75), a prominent positive deflection (P100), and a later negative deflection (N135). The peak latency and peak to peak amplitudes of these waves are measured (Mishra and Kalita, 2004). N75 reflects the activity of fovea and primary visual cortex. P100 originates from dorsal and ventral extrastriate cortex and represents the processing of stimulus characteristics and visuospatial selection (Di Russo et al., 2005; Hamilton et al., 2020). The latency and amplitude of N75 and P100 were calculated in this study.

\section{Flash Electroretinogram}

All participants underwent the FERG examinations using LKC UTAS-E 3000 system (LKC Technologies, Inc., Gaithersburg, USA). The white flash illumination was provided by Ganzfeld 2503D stimulator (LKC Technologies, Inc., Gaithersburg, MD, USA). The software LKC EM for Windows (EMwin v3.0; LKC Technologies, Inc., Gaithersburg, MD, USA) was used to control the recording setting and analyze the data. Signal amplification, luminance calibration, and bandpass filtering were integrated into the LKC system. Standard Ganzfeld scotopic (darkadapted) and photopic (light- adapted) ERGs were obtained by specialists after pupillary dilation. Subjects took $10 \mathrm{~min}$ of light adaptation before recording light-adapted ERGs and were dark adapted for at least 30 min before dark-adapted ERGs were obtained. Topical anesthesia was used for contact lens electrodes. Electroretinographic waveforms were simultaneously obtained from each eye by positioning the active electrodes (ERG-jet monopolar contact lens electrodes; UniversoPlastique SA, Le Cret-Du-Locle, Switzerland) on each cornea. The reference and ground electrodes (Grass subdermal needle electrode; AstroMed, Inc., West Warwick, RI) were placed subcutaneously in the pinna.

Three ERG recordings were obtained per stimulus intensity. The scotopic and photopic white flash stimuli were performed with an intrastimulus interval of $1 \mathrm{~min}$. The intrastimulus background recovery interval allowed the retina to recover from the previous flash. Photopic tests included: a $100 \mathrm{Td}-\mathrm{s}(\sim 4 \mathrm{~cd}-$ $\mathrm{s} / \mathrm{m} 2$ [assuming a $6 \mathrm{~mm}$ pupil diameter]) flash stimulus presented at a $1 \mathrm{~Hz}$ repetition rate with no background luminance (P1); a $58 \mathrm{Td}$-s ( $2 \mathrm{~cd}-\mathrm{s} / \mathrm{m} 2$ [assuming a $6 \mathrm{~mm}$ pupil diameter]) red 
stimulus presented at $3.4 \mathrm{~Hz}$, with a $380 \mathrm{Td}$ blue background (P2); a 100 Td-s ( $\sim 4 \mathrm{~cd}-\mathrm{s} / \mathrm{m} 2$ [assuming a $6 \mathrm{~mm}$ pupil diameter]) flash stimulus presented at a $2 \mathrm{~Hz}$ repetition rate with a 340 Td background (P3); and an 85 Td-s $(\sim 3 \mathrm{~cd}-\mathrm{s} / \mathrm{m} 2$ [assuming a $6 \mathrm{~mm}$ pupil diameter]) flickering (at $28.3 \mathrm{~Hz}$ ) stimulus (PF). For scotopic tests, stimulus intensity increased by a factor of 10 for each trial, beginning with a $2.8 \mathrm{Td}-\mathrm{s}(\sim 0.10 \mathrm{~cd}-\mathrm{s} / \mathrm{m} 2$ [assuming a $6 \mathrm{~mm}$ pupil diameter]) flash stimulus presented at $0.25 \mathrm{~Hz}(\mathrm{~S} 1)$, then a $28 \mathrm{Td}-\mathrm{s}(\sim 1 \mathrm{~cd}-\mathrm{s} / \mathrm{m} 2$ [assuming a $6 \mathrm{~mm}$ pupil diameter]) flash stimulus presented at $0.1 \mathrm{~Hz}$ (S2), and finally, a $280 \mathrm{Td}$-s ( $\sim 10 \mathrm{~cd}-\mathrm{s} / \mathrm{m} 2$ [assuming a $6 \mathrm{~mm}$ pupil diameter]) flash stimulus presented at $0.05 \mathrm{~Hz}$ (S3). Flicker ERG was obtained under the same conditions of light adaptation as the light-adapted ERG. And flashes were presented at a rate of $\sim 30$ stimuli per second.

\section{Magnetic Resonance Imaging}

MRI Scans were performed on a 3.0T EXCITE HD MR imaging system (Echo-speed plus, General Electric, Milwaukee, WI, USA) with an 8NVHEAD-A coil using a three- dimensional, spoiled gradient recalled echo 3D-SPGR-T1 weighted sequence using the following parameters: repetition time $(\mathrm{TR})=7.7 \mathrm{~ms}$; echo time $=1.6 \mathrm{~ms}$; flip angle $=15^{\prime}$; number of excitations $=1$; section thickness $=0.5 \mathrm{~mm}$; field of view $=240 \times 240 \mathrm{~mm}$; and matrix size $=256 \times 256 \mathrm{~mm}$. The voxel size was $1 \times 1 \times 1 \mathrm{~mm}$. The voxel-based morphometry was used to analyze the hippocampal volume, with the Biological Parametric Mapping (WFU PickAtlas Tool, http://fmri.wfubmc.edu/) and the MATLAB platform (version 7.0, Mathworks Inc. Sherbom, MA, USA) was used to calculate the hippocampal structure volume.

\section{Statistical Analysis}

Both eyes for each subject were examined and the average of these measurements was taken for the both eyes. Hippocampal volumes of both sides were obtained and the mean value of both sides was included in the analysis. Statistical analysis was performed using SPSS Version 26.0 (SPSS Inc., Chicago, IL, USA) and $P<0.05$ was considered significant. One-way ANOVAs were used to compare group differences in demographic and clinical variables between the three groups $(\mathrm{AD}, \mathrm{MCI}$, and cognitively normal control). We used chi-squared and split chi-squared tests to identify differences in sex and APOE 4 carrier status between the three groups. The Pearson correlation was used to determine the associations between hippocampal volume and ocular indexes, while the partial correlation coefficient $(r)$ and associated probability $(P)$ were calculated using partial correlation analysis after adjusting for confounding factors, such as age, sex, and years of education. We performed a Bonferroni correction by dividing the critical $P$-value by the number of comparisons being made. For multiple comparisons of demographic characteristics, ocular measurements and hippocampus volumes among $\mathrm{AD}, \mathrm{MCI}$, and cognitively normal group, the adjusted $P$-value required for significance is $0.05 / 3=0.017$. For correlation analysis, $P$-value is adjusted at $0.05 / 5=0.01$. We conducted hierarchical multiple stepwise linear regression analysis to evaluate the possible factors affecting the scores of ADAS-cog, MMSE and MoCA, including age, sex, years of education, APOE 4 carrier status, ophthalmologic measurements and hippocampal
TABLE 1 | Demographic characteristics of all participants.

\begin{tabular}{lcccc}
\hline Demographics & NC & MCI & AD & $P$-value \\
\hline N & 19 & 23 & 17 & - \\
Age, y, (SD) & $66.63(6.17)$ & $68.43(5.70)$ & $70.24(7.53)$ & 0.251 \\
Sex M/F & $8 / 11$ & $12 / 11$ & $9 / 8$ & 0.756 \\
Education, y, (SD) & $10.74(3.00)$ & $12.78(3.48)$ & $10.47(3.47)$ & 0.057 \\
ApoE4 carrier (\%) & $15.8 \%$ & $30.4 \%$ & $41.2 \%$ & 0.238 \\
MMSE (SD) & $28.79(1.03)$ & $26.91(1.47)^{\#}$ & $21.18(3.09)^{*} 8$ & $<0.001$ \\
MoCA (SD) & $24.89(2.13)$ & $20.57(2.21)^{\#}$ & $15.65(2.81)^{*} 8$ & $<0.001$ \\
ADAS-cog (SD) & $14.21(4.57)$ & $18.00(3.22)^{\#}$ & $31.88(4.31)^{+8}$ & $<0.001$ \\
SAS (SD) & $28.26(5.11)$ & $27.26(4.87)$ & $27.64(4.78)$ & 0.789 \\
SDS (SD) & $27.47(6.44)$ & $29.96(6.15)$ & $29.76(6.10)$ & 0.389 \\
\hline
\end{tabular}

$A D$, Alzheimer's disease; $M C l$, mild cognitive impairment; NC, normal control; $M$, male; $F$, female; y, years; SD, standard deviation; MMSE, Mini-Mental State Examination; MoCA, Montreal Cognitive Assessment; ADAS-cog, Alzheimer's Disease Assessment Scalecognitive subscale; SAS, Self-rating anxiety scale; SDS, Self-rating depression scale.

${ }^{\#} \mathrm{MCl}$ vs. NC, $P<0.017$.

${ }^{*} A D$ vs. $M C l, P<0.017$

$\S_{A}$ V vs. NC, $P<0.017$.

volumes. For each model, the regression coefficients $(\beta)$, R-squared $\left(R^{2}\right)$, change of $\mathrm{R}^{2}\left(\Delta R^{2}\right)$, and change of $F(\Delta F)$ were calculated, respectively.

\section{RESULTS}

In this research, 19 cognitively normal controls, $23 \mathrm{MCI}$ patients and $17 \mathrm{AD}$ patients were included. There was no significant difference in age, sex, years of education and APOE 4 carrier status among three groups. MMSE, MoCA and ADAS-cog were performed to assess cognitive function for all participants, and the scores of which revealed significantly typical trends among groups as expected $(P<0.001)$. Detailed demographic characteristics of each group are shown in Table $\mathbf{1}$.

Results of ocular measurements are listed in Table 2. Of the PVEP waveform components recognized, AD patients showed a decrease in the P100 amplitudes when compared to MCI group $(P=0.016)$, while comparison of the N75 wave revealed no statistically significant differences for the amplitudes or the latencies. In the FERG tests, eyes of AD patients had significantly prolonged rod response latency time when observed in the dark-adapted environment in comparison with age-matched cognitively normal controls $(P=0.013)$. As light stimulus in darkness was intensified to activate the cone system and mixed responses from both rod and cone systems were recorded, delayed rod cone response latency time was found in both MCI $(P=0.003)$ and $\mathrm{AD}(P=0.003)$ patients compared to normal controls. In the light environment, where responses from the cone system were recorded, $\mathrm{AD}$ patients showed longer reaction time reflected as increased 3.0 flicker latencies when compared to MCI patients $(P=0.013)$ and healthy participants $(P=0.008)$. However, no statistically significant group difference was found in amplitudes of all responses. In addition, structural changes were examined by OCT tests. Remarkably reduced thickness of $\mathrm{m}$-RNFL was observed in $\mathrm{AD}$ eyes than that in MCI eyes 
TABLE 2 | PVEP, FERG, OCT results, and Hippocampal volumes for all participants.

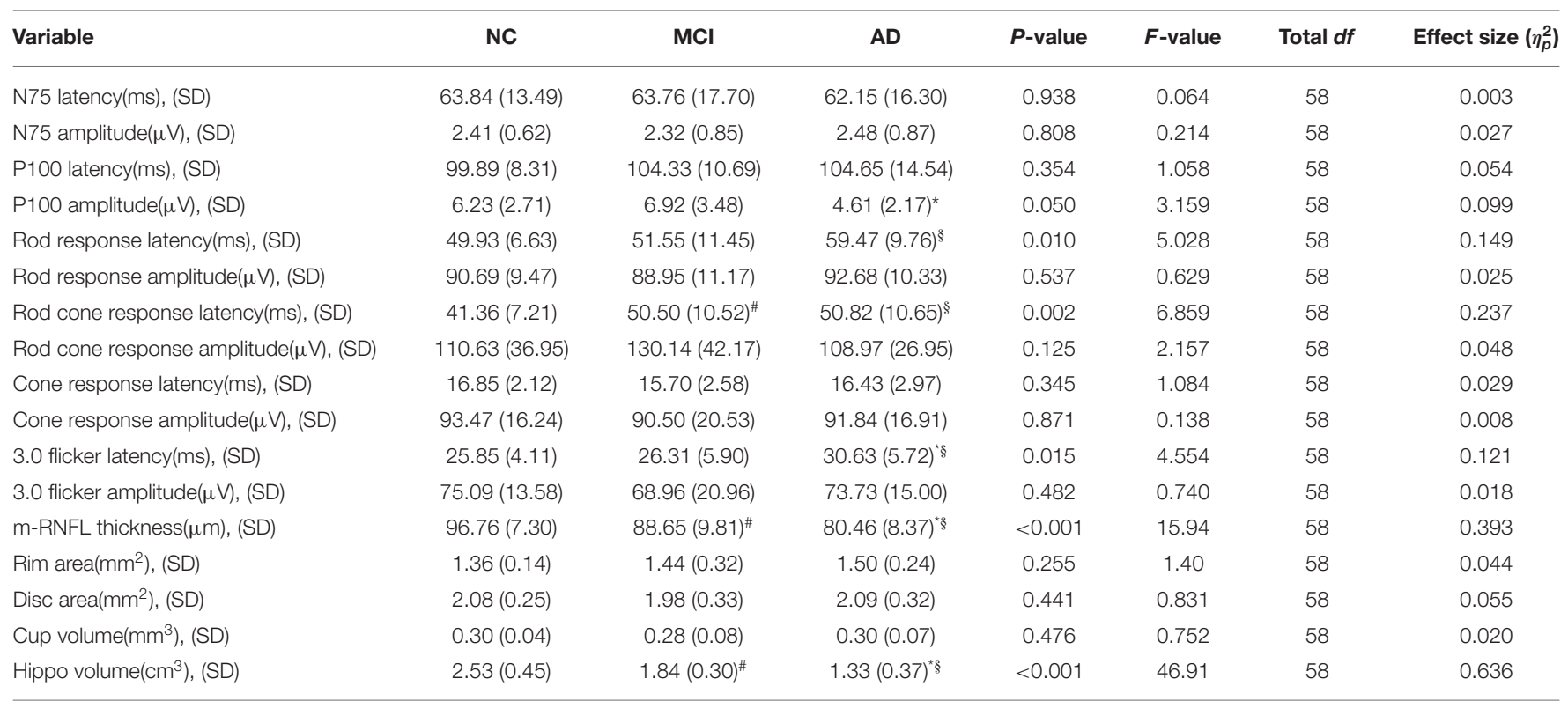

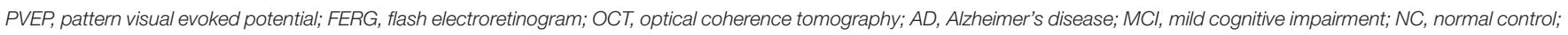
$S D$, standard deviation; $m$-RNFL, macular retinal nerve fiber layer; Hippo, hippocampus.

${ }^{\#} \mathrm{MCl}$ vs. NC, $P<0.017$.

${ }^{\star} A D$ vs. $M C l, P<0.017$.

${ }^{\S} A D$ vs. NC, $P<0.017$
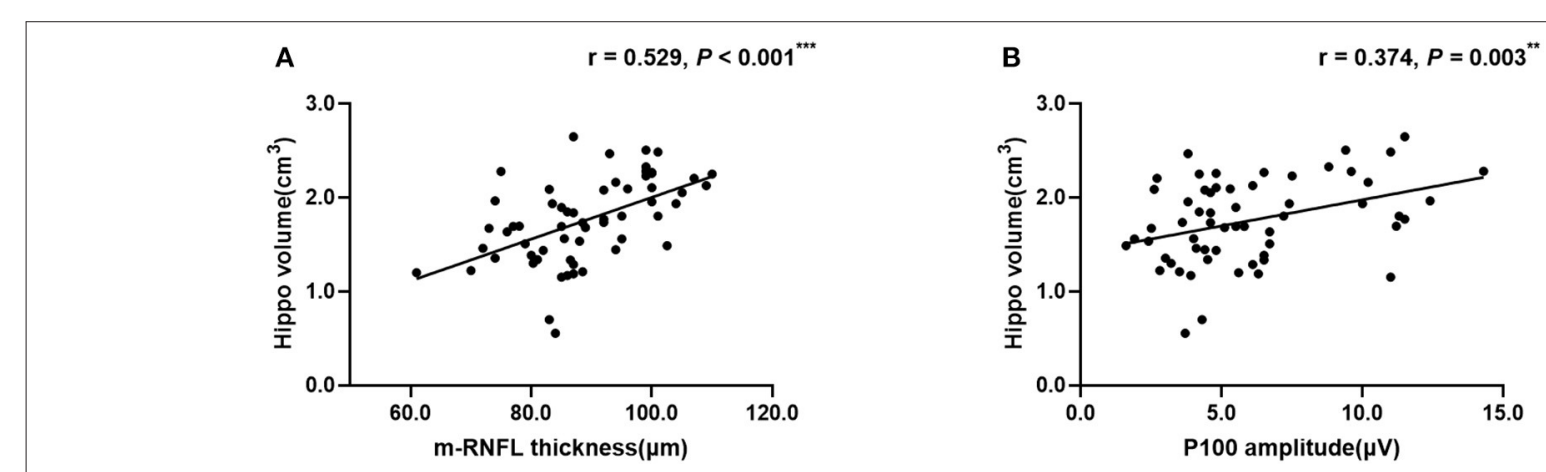

FIGURE 1 | Correlation analysis between hippocampal volume and m-RNFL thickness (A) or P100 amplitude (B). The parameter was estimated using Pearson's correlation coefficients. Hippo, hippocampus; m-RNFL, macular retinal nerve fiber layer. ${ }^{\star \star \star} P<0.001,{ }^{\star \star} P<0.01$.

$(P=0.014)$ and $\mathrm{NC}$ eyes $(P<0.001)$. MCI patients showed significantly thinner $\mathrm{m}$-RNFL compared to normal individuals $(P=0.011)$ as well. However, no significant morphological changes in the optic disc head were found for the rim area, disc area, and cup volume.

Regarding hippocampal volumes investigated by MRI, as shown in Table 2, significantly decreased size of hippocampus was found in patients with $\mathrm{AD}$ when compared to MCI group $(P<0.001)$ and healthy individuals $(P<0.001)$. A comparison of the MCI group with the NC group showed obviously shrunken structure of hippocampus as well $(P<0.001)$. Then we performed Pearson correlation analysis to explore associations between hippocampal volumes and ocular abnormalities as described in PVEP, FERG, and OCT examinations, respectively.
As shown in Figure 1, m-RNFL thickness $(r=0.529, P<$ 0.001 , Figure 1A) and P100 amplitude $(r=0.374, P=0.003$, Figure 1B) significantly correlated with hippocampal volumes. With adjustments made for the confounding factors of age, sex, years of education and $A P O E 4$ carrier status, as shown in Table 3, associations remained significant between the volumes of hippocampus and m-RNFL thickness $(r=0.521, P<0.001)$ as well as P100 amplitude $(r=0.389, P=0.003)$. We did not find any significant associations between rod response latency, rod cone response latency, 3.0 flicker latency and the brain structure of hippocampus.

A hierarchical regression analysis was further conducted to determine the associations between ocular indexes, hippocampal volumes and cognitive function. After controlling for age, sex, 
TABLE 3 | Partial correlation analysis between visual indexes and hippocampal volume.

\begin{tabular}{lcc}
\hline Variable & $\boldsymbol{r}$-value & $\boldsymbol{P}$-value \\
\hline m-RNFL thickness & 0.521 & $<0.001^{*}$ \\
P100 amplitude & 0.389 & $0.003^{*}$ \\
Rod response latency & -0.183 & 0.178 \\
Rod cone response latency & 0.078 & 0.570 \\
3.0 flicker latency & -0.065 & 0.634
\end{tabular}

m-RNFL, macular retinal nerve fiber layer.

The partial correlation coefficient of the correlation analysis was corrected with confounding factors including age, gender, and years of education.

${ }^{\star} P<0.01$.

TABLE 4 | Hierarchical regression analysis for ADAS-cog score in all participants.

\begin{tabular}{lccc}
\hline Variable & Regression 1 & Regression 2 & Regression 3 \\
\hline Age & 0.141 & 0.032 & -0.061 \\
Gender & -0.068 & -0.147 & -0.111 \\
Years of education & -0.205 & -0.219 & -0.167 \\
ApoE4 status & 0.146 & 0.090 & 0.083 \\
Hippo volume & & $-0.667^{\star \star \star}$ & $-0.490^{\star \star \star}$ \\
m-RNFL thickness & & & $-0.242^{\star}$ \\
P100 amplitude & & & -0.028 \\
Rod response latency & & & 0.204 \\
Rod cone response latency & & & 0.044 \\
3.0 flicker latency & & 0.528 & 0.173 \\
$R^{2}$ & 0.107 & 0.421 & 0.635 \\
$\Delta R^{2}$ & 0.107 & $47.262^{\star \star \star}$ & 0.106 \\
$\Delta F$ & 1.625 & & $2.794^{\star}$
\end{tabular}

ADAS-cog, Alzheimer's Disease Assessment Scale-cognitive subscale; Hippo, hippocampus; $m$-RNFL, macular retinal nerve fiber layer.

${ }^{\star \star \star} P<0.001,{ }^{\star} P<0.05$.

years of education and $A P O E 4$ carrier status, hippocampal volumes $(\beta=-0.490, P<0.001)$ and m-RNFL thickness $(\beta$ $=-0.242, P=0.031)$ were significantly associated with ADAScog scores (Table 4). Regarding scores of MMSE (Table 5) and MoCA (Table 6), however, just the parameter of hippocampal volumes demonstrated strongly positive associations ( $\beta=0.527$, $P<0.001 ; \beta=0.664, P<0.001$; respectively), while parameters of ocular indexes revealed no significant association $(P>0.05)$.

\section{DISCUSSION}

This study performed ophthalmic measurements including PVEP, FERG and OCT tests to investigate functional and structural changes of retina and/or visual pathway in MCI and $\mathrm{AD}$ patients compared to cognitively normal aging. Our results demonstrated that PVEP amplitude of P100 waveform was significantly decreased in $\mathrm{AD}$ patients compared to MCI and normal individuals. In FERG test, delayed latencies of rod response, rod cone response and 3.0 flicker time were found in cognitively impaired groups, indicating dysfunctions of both the rod, and cone systems in the disease
TABLE 5 | Hierarchical regression analysis for MMSE score in all participants.

\begin{tabular}{lccc}
\hline Variable & Regression 1 & Regression 2 & Regression 3 \\
\hline Age & -0.006 & 0.095 & 0.219 \\
Gender & -0.113 & -0.040 & -0.054 \\
Years of education & 0.218 & 0.230 & 0.159 \\
ApoE4 status & -0.188 & -0.136 & -0.139 \\
Hippo volume & & $0.617^{\star * \star}$ & $0.527^{\star * \star}$ \\
m-RNFL thickness & & & 0.134 \\
P100 amplitude & & & -0.004 \\
Rod response latency & & & -0.177 \\
Rod cone response latency & & & -0.128 \\
3.0 flicker latency & & & -0.022 \\
$R^{2}$ & 0.076 & 0.437 & 0.491 \\
$\Delta R^{2}$ & 0.076 & 0.361 & 0.054 \\
$\Delta F$ & 1.115 & $33.942^{* * *}$ & 1.024
\end{tabular}

MMSE, Mini-Mental State Examination; Hippo, hippocampus; m-RNFL, macular retinal nerve fiber layer.

${ }^{* \star *} P<0.001$

TABLE 6 | Hierarchical regression analysis for MoCA score in all participants.

\begin{tabular}{lccc}
\hline Variable & Regression 1 & Regression 2 & Regression 3 \\
\hline Age & -0.041 & 0.068 & 0.068 \\
Gender & -0.078 & 0.001 & -0.075 \\
Years of education & 0.007 & 0.021 & 0.046 \\
ApoE4 status & -0.191 & -0.135 & -0.108 \\
Hippo volume & & $0.664^{\star * *}$ & $0.664^{\star * \star}$ \\
m-RNFL thickness & & & 0.085 \\
P100 amplitude & & & -0.170 \\
Rod response latency & & & -0.050 \\
Rod cone response latency & & & 0.025 \\
3.0 flicker latency & & & -0.123 \\
$R^{2}$ & 0.042 & 0.460 & 0.506 \\
$\Delta R^{2}$ & 0.042 & 0.417 & 0.047 \\
$\Delta F$ & 0.597 & $40.957^{\star * *}$ & 0.905
\end{tabular}

MoCA, Montreal Cognitive Assessment; Hippo, hippocampus; $m$-RNFL, macular retinal nerve fiber layer.

${ }^{* \star *} P<0.001$

progression. OCT test revealed reduced m-RNFL thickness in $\mathrm{MCI}$ and $\mathrm{AD}$ patients, which significantly correlated with brain structure of hippocampus particularly vulnerable during the progression of AD. Strikingly, P100 amplitude showed significant association with hippocampal volumes even after adjusting confounding factors including age, sex, and years of education. Hierarchical regression analysis further demonstrated that mRNFL thickness, as well as hippocampal volumes, had significant predictive value in assessing cognitive performance in terms of neuropsychological test of ADAS-cog.

PVEPs are used to evaluate the functional integrity of the visual pathway. P100, typically peaking at about $100 \mathrm{~ms}$, is the major component of the PVEPs. It is considered to generate from dorsal and ventral extrastriate cortex and represent the 
processing of stimulus characteristics and visuospatial selection (Di Russo et al., 2005). Extrastriate cortex belongs to part of visual association areas and shows significant microscopic pathology with beta-amyloid (A $\beta$ ) aggregates and neurofibrillary tangles in the post-mortem investigation of $\mathrm{AD}$ patients (Arnold et al., 1991). High synaptic complexity of the association cortices may enable and amplify the propagation of disease pathology (Mckee et al., 2006). In line with previous reports (Krasodomska et al., 2010; Stothart et al., 2015), we propose that reduced $\mathrm{P} 100$ amplitude in $\mathrm{AD}$ patients may reflect this pathology in visual association areas and prove a sensitive marker in examining cortical pathology in $\mathrm{AD}$ progression beyond currently available behavioral, imaging and biochemical tools. Interestingly, partial correlation analysis revealed that decreased P100 amplitude significantly positively correlated with atrophied volume of hippocampus. Recent findings suggest that hippocampus participates in visuospatial memory formation. Dysfunction of synaptic plasticity in the visual cortex may influence visuospatial information process and thus in turn hippocampal formation (Tsanov and Manahan-Vaughan, 2008). Close relationship between P100 amplitude and hippocampal volumes may strengthen the potential value of PVEP waveform of P100 in early screening and monitoring the disease progression.

FERG provides an objective measure of cellular function in retina. The cellular responses recorded can be specific to rod photoreceptors under dark-adapted conditions or cone photoreceptors in light-adapted environment. In our study, prolonged latencies of rod response and 3.0 flicker time were found in $\mathrm{AD}$ patients when compared to MCI group and/or normal aging, while delayed rod cone response latency was observed in both MCI and AD patients compared to healthy controls, indicative of being a potential marker in early stage of cognitive decline. In an animal study of mice carrying APOE4, the most prevalent genetic risk factor for $\mathrm{AD}$, ERG recordings revealed significant attenuation of mixed rod cone responses in dark-adapted eyes, which is considered to be related to observed decrease in synaptic density of the retinal synaptic layers (Ran et al., 2013). However, our following correlation analysis demonstrated no significant association between FERG components and hippocampal volumes. In view of recent reports of FERG alterations in other neurodegenerative diseases (Devos et al., 2005; Pearl et al., 2017), more comprehensive longitudinal studies are needed to improve its specificity in clinical practice.

Retina has been viewed as an extension of central nervous system due to their developmental and structural similarities. Histopathological studies reported typical $A \beta$ deposits, loss of retinal ganglion cells and optic nerve degeneration in $\mathrm{AD}$ patients, hence initiating in the retrograde damaged ganglion cell fibers and leading to the morphological changes, such as RNFL thinning (Blanks et al., 1996a,b). Consistent with recent evidence (den Haan et al., 2017b; Chan et al., 2018), we have found significantly reduced overall thickness of $\mathrm{m}$ $\mathrm{RNFL}$ in $\mathrm{AD}$ and $\mathrm{MCI}$ patients compared to age-matched control counterparts in the current research. In addition, our results demonstrated a significant correlation between total $\mathrm{m}$ RNFL thickness and hippocampal volumes, corroborating the link between retina and brain in the progression of cognitive impairment. Several studies have investigated the connections between retinal changes and MRI volumetric measurements of brain structures. In early-onset $\mathrm{AD}$ patients, den Haan et al. (2017a) found total macular thickness correlated with parietal cortical atrophy, suggestive of reflection of cerebral cortical changes in the retina, independent of $\mathrm{AD}$ pathological markers, such as amyloid. In non-demented older adults, thinner overall RNFL and peripapillary RNFL showed good associations with smaller volumes of medial temporal lobes and hippocampus, while no significant associations were found in the control regions, which were located outside the visual pathway and the regions involved in AD (Méndez-Gómez et al., 2018; Shi et al., 2020). In a recent study involving AD and MCI patients, better correlation was observed between inner perifovea retinal thickness and the hippocampal and entorhinal cortical volumes, which are specifically affected in the early stage of $\mathrm{AD}$, although no correlation was found between $\mathrm{m}$ RNFL thickness and cognitive performance, and no significant difference of $\mathrm{m}$-RNFL thinning was shown in patients with MCI compared to those with AD (Tao et al., 2019). In our study, m-RNFL thickness exhibited a significant reduction in MCI patients in comparison to $\mathrm{AD}$ patients and presented as an independent predictor of ADAS-cog score, along with average hippocampal volumes, in the hierarchical regression model. The discrepancies may result from high degree of variability in the study inclusion/exclusion criteria, assessment of neuropsychological performance and the rigor of adjustment of confounding factors. However, our results have added more evidence for the availability of application of m-RNFL thickness as a promising biomarker for future $\mathrm{AD}$ diagnosis, monitoring, and prognosis.

In the current cross-sectional study we have enrolled relatively small sample size, partially due to strict exclusion criteria of patient enrollment and adaptability to full screen of ocular structural and functional measurements. A longitudinal study with expanded sample size is required to validate the retinabrain association in the neurodegenerative process of cognitive decline. Owing to the lack of $A \beta$ or Tau-related markers, we were unable to analyze the relationship between those pathological markers and ocular measurements, and hence the present findings cannot directly confirm whether the alterations in the ophthalmological examinations are specific to AD. Additionally, quandrant-specific changes of peripapillary RNFL thickness have been reported in recent studies, although results remained controversial (Alber et al., 2020). Evidence from numerous neuropsychological and functional neuroimaging studies suggested that right posterior hippocampus is implicated in visual information and visuospatial memory formation. Changes in the volume of the anterior and posterior hippocampus can be compensated and therefore underestimate the alteration of the hippocampus most involved in vision (Hüfner et al., 2011). Braak's staging of AD showed that neurodegeneration began in entorhinal and perirhinal cortex suggesting the area most and earliest affected by neurofibrillary tangles in Alzheimer's disease, even before the hippocampus (Braak and Braak, 1995). Further detailed studies with investigations of 
changes of different segmentation of RNFL thickness and more brain regions including entorhinal cortex, perirhinal cortex and subareas of hippocampus are needed to assist in clarification of possible mechanisms underlying retina-brain association in the disease progression of cognitive decline and $\mathrm{AD}$.

In conclusion, our results presented significant alterations in ophthalmological examinations including PVEP, FERG responses and $\mathrm{m}$-RNFL thickness in patients with MCI and AD. P100 amplitude and m-RNFL thickness showed significant correlations with brain structure involved in $\mathrm{AD}$-related neurodegeneration, and therefore proved to be potential indicators of brain imaging pathologies. Future prospective researches are required to determine the reliability of ocular investigations, especially measurement of RNFL thickness during AD progression and recognize whether retina has broad implications in $\mathrm{AD}$ pathology to serve as a promising non-invasive and cost-effective biomarker.

\section{DATA AVAILABILITY STATEMENT}

The raw data supporting the conclusions of this article will be made available by the authors, without undue reservation.

\section{REFERENCES}

Alber, J., Goldfarb, D., Thompson, L. I., Arthur, E., Hernandez, K., Cheng, D., et al. (2020). Developing retinal biomarkers for the earliest stages of Alzheimer's disease: what we know, what we don't, and how to move forward. Alzheimers Dement. 16, 229-243. doi: 10.1002/alz.12006

Armstrong, R. A. (1996). Visual field defects in alzheimer's disease patients may reflect differential pathology in the primary visual cortex. Optom Vis Sci. 73:677-682. doi: 10.1097/00006324-199611000-00001

Arnold, S. E., Hyman, B. T., Flory, J., Damasio, A. R., and Hoesen, G. W. V. (1991). The topographical and neuroanatomical distribution of neurofibrillary tangles and neuritic plaques in the cerebral cortex of patients with Alzheimer's disease. Cereb. Cortex 1, 103-116. doi: 10.1093/cercor/1.1.103

Blanks, J. C., Schmidt, S. Y., Torigoe, Y., Porrello, K. V., Hinton, D. R., and Blanks, R. H. (1996a). Retinal pathology in Alzheimer's disease. II. Regional neuron loss and glial changes in GCL. Neurobiol. Aging 17, 385-395. doi: 10.1016/0197-4580(96)00009-7

Blanks, J. C., Torigoe, Y., Hinton, D. R., and Blanks, R. H. (1996b). Retinal pathology in Alzheimer's disease. I. Ganglion cell loss in foveal/parafoveal retina. Neurobiol. Aging 17, 377-384. doi: 10.1016/0197-4580(96)0 0010-3

Braak, H., and Braak, E. (1995). Staging of Alzheimer's disease-related neurofibrillary changes. Neurobiol. Aging 16, 271-278; discussion 278-84. doi: 10.1016/0197-4580(95)00021-6

Chan, K. Y., Campbell, H., Wang, W., Rudan, I., Wang, W., Wu, J. J., et al. (2013). Epidemiology of Alzheimer's disease and other forms of dementia in China, 1990-2010:a systematic review and analysis. Lancet 381, 2016-2023. doi: 10.1016/S0140-6736(13)60221-4

Chan, V. T. T., Sun, Z., Tang, S., Chen, L. J., Wong, A., Tham, C. C., et al. (2018). Spectraldomain OCT measurements in Alzheimer's disease: a systematic review and meta analysis. Ophthalmology 126, 497-510. doi: 10.1016/j.ophtha.2018.08.009

Cormack, F. K., Tovee, M., and Ballard, C. (2000). Contrast sensitivity and visual acuity in patients with Alzheimer's disease. Int. J. Geriatr. Psychiatry 15, 614-620. doi: 10.1002/1099-1166(200007)15:7<614::AID-GPS153>3.0.CO;2-0

\section{ETHICS STATEMENT}

The studies involving human participants were reviewed and approved by Ruijin Hospital Ethics Committee, Shanghai Jiao Tong University School of Medicine. The patients/participants provided their written informed consent to participate in this study.

\section{AUTHOR CONTRIBUTIONS}

YD and WX designed the study, edited the manuscript, and validated the statistics. AZ and FF collected the data, wrote and edited the manuscript, and performed the statistics. YC and YW collected the data and help revised the manuscript. BL and YQ collected the data. All authors contributed to the article and approved the submitted version.

\section{FUNDING}

This study was supported by grants from the National Natural Science Foundation of China (No. 81571029) and the Natural Science Foundation of Shanghai (No. 19ZR1432500) to YD; the Shanghai Municipal Commission of Health and Family Planning (No. 201640042) to WX; the National Natural Science Foundation of China (No. 81801261) to FF.

Croningolomb, A. (1995). Vision in Alzheimer's disease. Gerontologist 35, 370-376. doi: 10.1093 /geront/35.3.370

den Haan, J., Janssen, S. F., van de Kreeke, J. A., Scheltens, P., Verbraak, F. D., and Bouwman, F. H. (2017a). Retinal thickness correlates with parietal cortical atrophy in early-onset Alzheimer's disease and controls. Alzheimers Dement. 10, 49-55. doi: 10.1016/j.dadm.2017.10.005

den Haan, J., Verbraak, F. D., Visser, P. J., and Bouwman, F. H. (2017b). Retinal thickness in Alzheimer's disease: a systematic review and meta-analysis. Alzheimers Dement. 25, 162-170. doi: 10.1016/j.dadm.2016.12.014

Devos, D., Tir, M., Maurage, C. A., Waucquier, N., Defebvre, L., DefoortDhellemmes, S., et al. (2005). ERG and anatomical abnormalities suggesting retinopathy in dementia with Lewy bodies. Neurology 65, 1107-1110. doi: 10.1212/01.wnl.0000178896.44905.33

Di Russo, F., Pitzalis, S., Spitoni, G., Aprile, T., Patria, F., Spinelli, D., et al. (2005). Identification of the neural sources of the pattern-reversal VEP. Neuroimage 24, 874-886. doi: 10.1016/j.neuroimage.2004.09.029

Dunstan, D. A., and Scott, N. (2018). Assigning clinical significance and symptom severity using the zung scales: levels of misclassification arising from confusion between index and raw scores. Depress. Res. Treat. 2018:9250972. doi: 10.1155/2018/9250972

Erten-Lyons, D., Howieson, D., Moore, M. M., Quinn, J., Sexton, G., Silbert, L., et al. (2006). Brain volume loss in MCI predicts dementia. Neurology 66, 233-235. doi: 10.1212/01.wnl.0000194213.50222.1a

Goldstein, F. C., Ashley, A. V., Miller, E., Alexeeva, O., Zanders, L., and King, V. (2014). Validity of the montreal cognitive assessment as a screen for mild cognitive impairment and dementia in African Americans. J. Geriatr. Psychiatry Neurol. 27, 199-203. doi: 10.1177/0891988714524630

Goldstein, L. E., Muffat, J. A., Cherny, R. A., Moir, R. D., Ericsson, M. H., Huang, X., et al. (2003). Cytosolic beta-amyloid deposition and supranuclear cataracts in lenses from people with Alzheimer's disease. Lancet 361, 1258-1265. doi: 10.1016/S0140-6736(03)12981-9

Hamilton, R., Bach, M., Heinrich, S. P., Hoffmann, M. B., Odom, J. V., McCulloch, D. L., et al. (2020). ISCEV extended protocol for VEP methods of estimation of visual acuity. Doc Ophthalmol. doi: 10.1007/s10633-02009780-1 
Heaton, G. R., Davis, B. M., Turner, L. A., and Cordeiro, M. F. (2015). Ocular biomarkers of Alzheimer's disease. Cent. Nerv. Syst. Agents Med. Chem. 15, 117-125. doi: 10.2174/1871524915666150319123015

Hüfner, K., Strupp, M., Smith, P., Brandt, T., and Jahn, K. (2011). Spatial separation of visual and vestibular processing in the human hippocampal formation. Ann. N. Y. Acad. Sci. 1233, 177-186. doi: 10.1111/j.1749-6632.2011.06115.x

Ikram, M. K., Cheung, C. Y., Wong, T. Y., and Chen, C. P. (2012). Retinal pathology as biomarker for cognitive impairment and Alzheimer's disease. J. Neurol. Neurosurg. Psychiatr. 83, 917-922. doi: 10.1136/jnnp-2011-301628

Iseri, P. K., Altina,ş, O., Tokay, T., and Yüksel, N. (2006). Relationship between cognitive impairment and retinal morphological and visual functional abnormalities in Alzheimer disease. J. Neuroophthalmol. 26, 18-24. doi: 10.1097/01.wno.0000204645.56873.26

Krasodomska, K., Lubiński, W., Potemkowski, A., and Honczarenko, K. (2010). Pattern electroretinogram (PERG) and pattern visual evoked potential (PVEP) in the early stages of Alzheimer's disease. Doc. Ophthalmol. 121, 111-121. doi: 10.1007/s10633-010-9238-x

Livingston, G., Huntley, J., Sommerlad, A., Ames, D., Ballard, C., Banerjee, S., et al. (2020). Dementia prevention, intervention, and care: 2020 report of the lancet commission. Lancet 396, 413-446. doi: 10.1016/S0140-6736(20)30367-6

Lopez, M. N., Charter, R. A., Mostafavi, B., Nibut, L. P., and Smith, W. E. (2005). Psychometric properties of the folstein mini-mental state examination. Assessment 12, 137-144. doi: 10.1177/1073191105275412

Mckee, A. C., Au, R., Cabral, H. J., Kowall, N. W., Seshadri, S., Kubilus, C. A., et al. (2006). Visual association pathology in preclinical Alzheimer disease. J. Neuropathol. Exp. Neurol. 65, 621-630. doi: 10.1097/00005072-200606000-00010

McKhann, G. M., Knopman, D. S., Chertkow, H., Hyman, B. T., Jack, C. R., Kawas, C. H., et al. (2011). The diagnosis of dementia due to Alzheimer's disease: recommendations from the national institute on aging-Alzheimer's association workgroups on diagnostic guidelines for Alzheimer's disease. Alzheimers Dement. 7, 263-269. doi: 10.1016/j.jalz.2011.03.005

Méndez-Gómez, J. L., Pelletier, A., Rougier, M. B., Korobelnik, J. F., Schweitzer, C., Delyfer, M. N., et al. (2018). Association of retinal nerve fiber layer thickness with brain alterations in the visual and limbic networks in elderly adults without dementia. JAMA Netw. Open. 1:e184406. doi: 10.1001/jamanetworkopen.2018.4406

Mishra, U. K., and Kalita, J. (2004). “Clinical neurophysiology,"in Visual Evoked Potential, 1st Edn, eds U. K. Mishra and J. Kalita (New Delhi: Elsevier), 249-266.

Odom, J. V., Bach, M., Barber, C., Brigell, M., Marmor, M. F., Tormene, A. P., et al. (2004). Visual evoked potentials standard. Doc. Ophthalmol. 108, 115-123. doi: 10.1023/B:DOOP.0000036790.67234.22

Ohno-Matsui, K. (2011). Parallel findings in age-related macular degeneration and Alzheimer's disease. Prog. Retin. Eye Res. 30, 217-238. doi: 10.1016/j.preteyeres.2011.02.004
Pearl, J. R., Heath, L. M., Bergey, D. E., Kelly, J. P., Smith, C., Laurino, M. Y., et al. (2017). Enhanced retinal responses in Huntington's disease patients. J. Huntingtons. Dis. 6, 237-247. doi: 10.3233/JHD-170255

Petersen, R. C. (2004). Mild cognitive impairment as a diagnostic entity. J. Intern. Med. 256, 183-194. doi: 10.1111/j.1365-2796.2004.01388.x

Petersen, R. C. (2006). Mild cognitive impairment. Lancet 367:1979. doi: 10.1016/S0140-6736(06)68881-8

Ran, A., Ezraelia, R., Weinberger, D., Solomon, A., Ofri, R., and Michaelson, D. M. (2013). ApoE4 induces synaptic and ERG impairments in the retina of young targeted replacement ApoE4 mice. PLoS ONE 8:e64949. doi: 10.1371/journal.pone.0064949

Shi, Z., Cao, X., Hu, J., Jiang, L., Mei, X., Zheng, H., et al. (2020). Retinal nerve fiber layer thickness is associated with hippocampus and lingual gyrus volumes in nondemented older adults. Prog. Neuropsychopharmacol. Biol. Psychiatry. 99:109824. doi: 10.1016/j.pnpbp.2019.109824

Stothart, G., Kazanina, N., Näätänen, R., Haworth, J., and Tales, A. (2015). Early visual evoked potentials and mismatch negativity in Alzheimer's disease and mild cognitive impairment. J. Alzheimers Dis. 44, 397-408. doi: 10.3233/JAD-140930

Tao, R., Lu, Z., Ding, D., Fu, S., Hong, Z., Liang, X., et al.(2019). Perifovea retinal thickness as an ophthalmic biomarker for mild cognitive impairment and early Alzheimer's disease. Alzheimers Dement. 11, 405-414. doi: 10.1016/j.dadm.2019.04.003

Tsanov, M., and Manahan-Vaughan, D. (2008). Synaptic plasticity from visual cortex to hippocampus: systems integration in spatial information processing. Neuroscientist 14, 584-597. doi: 10.1177/1073858408315655

Zainal, N. H., Silva, E., Lim, L. L., and Kandiah, N. (2016). Psychometric properties of Alzheimer's disease assessment scale-cognitive subscale for mild cognitive impairment and mild Alzheimer's disease patients in an Asian context. Ann. Acad. Med. Singap. 45, 273-283.

Zhang, M. Y., Katzman, R., Salmon, D., Jin, H., Cai, G. J., Wang, Z. Y., et al. (1990). The prevalence of dementia and Alzheimer's disease in Shanghai, China: impact of age, gender, and education. Ann. Neurol. 27, 428-437. doi: 10.1002/ana.410270412

Conflict of Interest: The authors declare that the research was conducted in the absence of any commercial or financial relationships that could be construed as a potential conflict of interest.

Copyright (c) 2021 Zhao, Fang, Li, Chen, Qiu, Wu, Xu and Deng. This is an openaccess article distributed under the terms of the Creative Commons Attribution License (CC BY). The use, distribution or reproduction in other forums is permitted, provided the original author(s) and the copyright owner(s) are credited and that the original publication in this journal is cited, in accordance with accepted academic practice. No use, distribution or reproduction is permitted which does not comply with these terms. 